\title{
Choosing the Right Technology to Support Your Business
}

\author{
John Eckhardt (Code Pros)
}

KEYWORDS: Professional, Scientific, Technical Services, Information, Software, Data, Information Technology.

Many business owners get excited about their product and market, but don't think much about the internal IT their business needs. This may be especially true if you're selling something that's not software intensive, such as pet toys or a lawncare service. But over time, the right technology will help your business run more smoothly, especially as it grows. It can help you fulfill orders, track inventory, streamline billing, simplify taxes, prevent costly mistakes and save hours of mindnumbing administrative work.

That's why it's important to plan for and take care of your business' tech needs. Here are several things to consider as you're thinking about how technology can support your business.

\section{Put the business before the technology}

Your business' purpose is fulfilling people's needs. The technology helping your business must support those goals, not the other way around. Your business, time and effort should not be going to support the technology infrastructure that you've become locked into. On the positive side, technology can help you fulfill people's needs faster, cheaper or better. For example, a bookstore can list its inventory online and sell to people in a larger market. The business stays the same while the technology helps it fulfill its mission better.

When business owners approach me with custom software ideas to help support their business, I frequently see one big problem: Their focus is on the underlying technology rather than their business. For example, they want something to run on Amazon Web Services with an Oracle database and it needs to have an API integration with Equifax. With a focus like this, the actual business purpose -- running a credit report and storing the system's decision on whether they're approved for a loan -- takes the back seat. In properly built software, the business process takes the front seat.

As a tech consultant, I feel the focus should always be: "What does this system do?"

A better way to describe the above system would be: "Users can provide data, which the system will use to run a credit report on them, and the system will use our formula to determine whether we should approve their loan or not." The business doesn't care about whether the software talks to Equifax or Experian. The business process doesn't care whether you're saving the data in an Oracle database or flat files.

With a solid software architecture, you can put the business process in the forefront. This also makes the system easier to change from one database to another in the future, and makes the software much more reliable. More reliable means it's cheaper for a business in the long run.

\section{Treat technology as an ongoing expense}

Think of the technology that you use to help run your business as a building that requires occasional maintenance. In your office or warehouse, you have ongoing costs like utilities and rent. "Technology" is likely to be a regular expense like these. It's wishful thinking to believe that you'll just buy one "superpowered" piece of software that's going to cover your tech needs for several years. Whether you pay a monthly fee for "software as a service" (SAAS) or pay up-front to have custom software crafted for your business, you can expect additional expenses from time to time.

As an example, imagine you manage your inventory with a spreadsheet. It's straightforward when you have five products, but when you add another category of products some change is necessary. Adding a new product line means adding a column, deciding how to fill in the missing old data, and training people to fill in the 
new data properly. That's a small example, but it illustrates the fact that change to a business process must then cascade down into your technology systems. Making that happen may require time and/or money.

A less desirable, but common, scenario is when some technology must change when the business doesn't change. This can happen when a security update to some part of the technology must happen, or when a software vendor stops supporting your favorite software version, causing upgrade costs and retraining. While the risk of this case can be reduced through properly designing your system and software, both changes should be budgeted for.

\section{Find the balance between current and future needs}

When your business is in its startup phase, your technology systems' needs are different from what you'll need after you've hired your first 100 employees. How do you make systems that work both now and in the future? Focus on your business, not the software that you're running. If you're in e-commerce, rather than focusing on online selling platforms such as Shopify or WooCommerce or Square, keep in mind that you're fulfilling orders to customers through a catalog. In the future of your business, you'll still be fulfilling orders through a catalog even if you're using e-commerce software for enterprises such as Magento (Adobe Commerce) or SAP.

If you write your process documents with that focus, you'll be able to switch from platform to platform by keeping your eyes on the main focus of fulfilling orders rather than the underlying technology. Software vendors often want to lock you into their product so that you continue to pay them money and can't leave, but keep your options open. Remember: technology is something that should serve your purposes; you shouldn't be tied or locked into a particular technology.

\section{Start Here}

If you haven't chosen technology to support your business yet, here are some things to think through:

- Write out "process documents" for each of your business processes, such as billing clients, building the product, receiving orders, shipping the product, hiring staff, etc. This involves making a checklist of every step for each of these processes. The better you understand how your business runs, the easier it will be to find or develop software to take it to the next level.

- Set aside time to identify and contact several possible business technology service providers in your area or country. Interview each one and make sure they're willing to talk to you about YOUR business needs, not just THEIR preferred software solutions. The best ones communicate clearly in language you can understand, not tech jargon.

- Talk to other small business owners you trust about how they've managed their technology needs. See if they are working with providers they're comfortable with or if they have problems like those described above, which you want to avoid. 